\title{
No effect of value learning on awareness and attention for faces: Evidence from continuous flash suppression and the attentional blink
}

Timo Stein ${ }^{1}$ and Sara Verosky ${ }^{2}$

${ }^{1}$ Brain and Cognition, Department of Psychology, University of Amsterdam, The Netherlands

${ }^{2}$ Department of Psychology, Oberlin College, United States of America

\section{Correspondence}

Timo Stein, Brain and Cognition, Department of Psychology, University of Amsterdam, 1001 NK Amsterdam, The Netherlands.

Email: timo@timostein.de 


\begin{abstract}
It is widely believed that the emotional and motivational value of social signals, such as faces, influences perception and attention. However, effects reported for stimuli with intrinsic affective value, such as emotional facial expressions, can often be explained by differences in low-level stimulus properties. To rule out such low-level effects, here we used a value-learning procedure, in which faces were associated with different probabilities of monetary gain and loss in a choice game. In three experiments involving 149 participants we tested the influence of affective valence (win- vs. loss-associated faces) and motivational salience (probability of monetary gain or loss) on visual awareness, attention, and memory. Using continuous flash suppression and rapid serial visual presentation we found no effects of affective valence or motivational salience on visual awareness of faces. Furthermore, in two experiments there was no evidence for a modulation of the attentional blink, indicating that acquired emotional and motivational value does not influence attentional priority of faces. However, we found that motivational salience boosted recognition memory, and this effect was particularly pronounced for win-associated faces. These results indicate that acquired affective valence and motivational salience affect only later processing of faces related to memory, but do not directly affect visual awareness and attention.
\end{abstract}




\section{Introduction}

The world contains myriad visual stimuli but only a limited amount of this information enters conscious awareness. One factor that can influence which stimuli receive attention and enter awareness is emotional and motivational value (Bourgeois, Chelazzi, \& Vuilleumier, 2016; Hedger, Gray, Garner, \& Adams, 2016). Much evidence for emotional prioritization has been acquired with continuous flash suppression (CFS), a strong interocular suppression technique (Tsuchiya \& Koch, 2005). Following initial suppression, stimuli with intrinsic emotional value, such as emotional facial expressions or emotional body postures break through CFS and become visible more quickly than non-emotional stimuli (Stein, 2019; Yang, Zald, \& Blake 2007; Zhan, Hortensius, \& De Gelder, 2015). Such effects are often considered evidence for unconscious processing of emotional stimuli, perhaps involving specialized neural circuitry (Tamietto \& De Gelder, 2010), resulting in privileged entry to perceptual awareness.

One issue for studies using stimuli with intrinsic emotional value is that these stimuli differ not only in emotional meaning but also in terms of lower-level image properties, such as shape, texture, phase, amplitude, and spatial frequency spectrum. Indeed, several effects are better explained by differences in such low-level properties than by differences in emotionalmotivational value (Gayet, Stein, \& Peelen, 2019; Gray et al., 2013; Moors et al., 2019). For example, shorter suppression times for fearful faces in CFS reflect higher effective contrast rather than enhanced processing of threat (Hedger, Adams, \& Garner, 2015). Similarly, the influence of facial dominance on breaking CFS is related to local contrast in the face's eye regions rather than to social evaluation (Stein, Awad, Gayet, \& Peelen, 2018). One elegant way of ruling out the influence of low-level stimulus properties is to use stimuli that differ only with regard to their affective learning history. For example, Gayet and colleagues (2016) found shorter suppression times for colored annuli that had been paired with electric shock in a fear conditioning procedure (Gayet, Paffen, Belopolsky, Theeuwes, \& Van der Stigchel, 2016). However, whether such effects of affective learning extend beyond conditioning of basic visual stimuli (colors, gratings, e.g. Padmala \& Pessoa, 2008) with threatening unconditioned stimuli (such as shocks) is currently unknown.

In our daily social lives, we routinely pair other people with affective information, such as value or affective semantic information such as emotional stories and episodes. However, two previous studies in which images of human faces were paired with affective information did not find an effect on breaking CFS (Rabovsky, Stein, \& Abdel Rahman, 2016; Stein, Grubb, 
Bertrand, Suh, \& Verosky, 2017). These studies used affective-learning protocols where participants learned to associate affective stories with particular face exemplars (e.g. negative story "Fired an employee before Christmas"). Thus, in contrast to the classical fear conditioning procedure by Gayet and colleagues (2016), affective learning involved semantic processing (associating affective stories with face exemplars). Furthermore, while in the classical fear conditioning paradigm stimuli were paired with a direct negative outcome for participants (a shock), faces were not. Here, we tested the influence of reinforcement learning on awareness of faces. Faces were associated with different probabilities of monetary gain and loss in a choice game, thus equipping the face stimuli with direct affective and motivational relevance for participants.

We adopted an established value-learning procedure, where faces were paired with different probabilities of monetary gain and loss. In previous studies using this choice game, expected value influenced visual processing of faces. For example, Raymond and O’Brien (2009) found distinct effects of motivational salience (probability of monetary gain or loss) and affective valence (gain or loss) on recognition memory in an attentional-blink (AB) paradigm. In the AB-task, participants indicated whether a face had previously been presented in the choice game. Participants were asked to identify a first stimulus, and faces followed this stimulus with a short or long temporal lag. At short lags face recognition suffered, reflecting reduced temporal attention characteristic of the AB. Faces with high motivational salience (high probability of an outcome in the choice game) were more often recognized correctly, independent of whether attention was fully available or reduced (long vs. short lags in the AB task). In contrast, affective valence influenced attentional priority, virtually eliminating the AB for faces that had been paired with monetary gain, indicating that "attention and motivation provide separable, independent top-down signals for controlling perceptual awareness" (Raymond \& O'Brien, 2009).

However, as this study measured how well participants could remember faces from the value-learning task, these effects could reflect differences in memory unrelated to perceptual awareness. The effect of motivational salience, which was independent of lag, could reflect differences in long-term memory or differences in response criterion and memory confidence. For example, for faces with higher motivational salience participants may have simply been more confident that they had seen the face before, rather than perceiving the face more clearly. 
The reduced $\mathrm{AB}$ for win-associated faces could similarly reflect memory prioritization rather than attentional enhancement of perceptual awareness.

Indeed, in line with the possibility that observed differences were due to differences in memory, other studies that tested the influence of value-learning on face processing but did not require memory retrieval yielded mixed results. For example, in an attentional cueing study targets were preceded by spatially congruent or incongruent face cues. At short cue-target stimulus onset asynchronies (SOAs) (100 ms), where one would expect effects of rapid spatial orienting, overall responses tended to be slower when win-associated faces were used as a cue, and there was no evidence for attentional cueing by faces with high motivational salience or positive valence (Rutherford, O’Brien, \& Raymond, 2010). Similarly, measuring perceptual discrimination of faces and scrambled faces with speeded saccades, Rothkirch and colleagues (2013) reported no evidence for faster detection of win- or loss-associated faces compared to neutral faces that had never been paired with a monetary outcome (Rothkirch, Ostendorf, Sax, \& Sterzer, 2013). Such absence of effects on attention and perception is consistent with an fMRI study that found differential activity in reward-related areas in orbitofrontal cortex following value learning but no modulation of visual cortex or fusiform face area (Rothkirch, Schmack, Schlagenhauf, \& Sterzer, 2012). Together, these results are difficult to reconcile with the idea that acquired value influences attentional priority and visual awareness of faces.

To test whether learned affective valence and motivational salience influence awareness of faces, in Experiment 1 we measured suppression times using breaking CFS (Jiang, Costello, \& He, 2007; Stein, 2019) for faces that differed in expected value following a value-learning task. To anticipate our results, although participants successfully learned to choose the optimal faces in the value-learning task, neither motivational salience nor affective value influenced awareness of faces during CFS. To determine whether this reflected the perceptual nature of our task (simple localization) or the fact that CFS abolished stimulus processing early in the visual system, we conducted two attentional-blink (AB) experiments. These experiments allowed us to distinguish between effects on visual awareness, attention, and memory. In Experiment 2a (AB-localization), participants localized faces embedded in a modified rapid serial visual presentation (RSVP) sequence. With this simple localization task, there was no evidence for effects of motivational salience or affective value on awareness and attention. Experiment $2 \mathrm{~b}$ (AB-recognition) was modeled after the study by Raymond and O'Brien (2009) but had substantially higher statistical power. Results revealed overall better recognition memory for 
faces with high motivational salience, in particular for win-associated faces, but no effects on attentional priority. Together, our results suggest that value associations for faces influence memory but not attention or awareness.

a

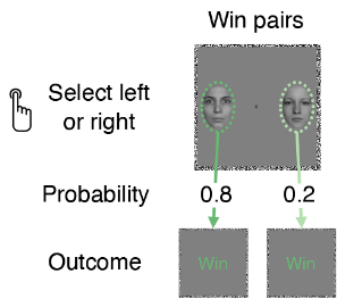

b

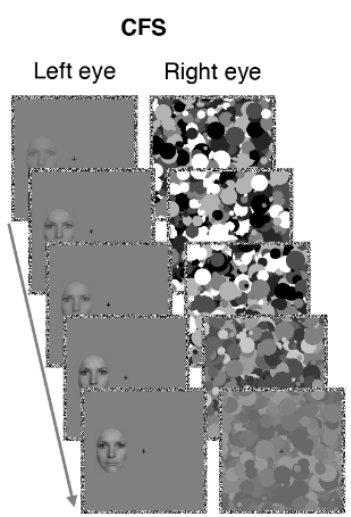

Speeded Ir localization
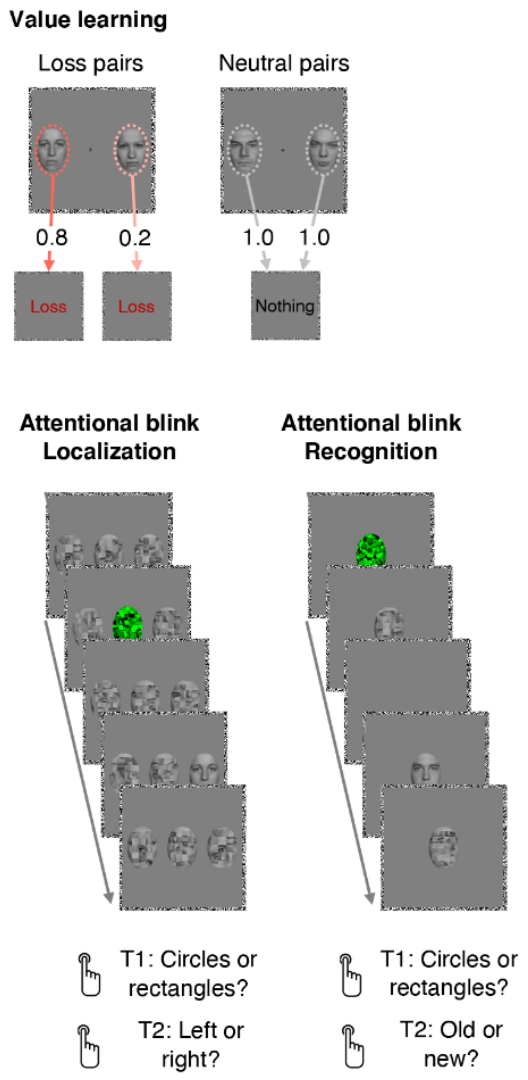

Figure 1. Schematics of the experiments. (a) In the value-learning task, participants were presented with pairs of faces, which could either lead to monetary gain, loss, or no outcome. Participants selected one face from each pair. One of the faces in rewarded and punished pairs was associated with high motivational salience (probability of win or loss $80 \%$ ), and the other with low motivational salience (probability of win or loss 20\%). (b) Example trials from Experiment 1 (continuous flash suppression [CFS] task) and from Experiment 2 (attentional-blink [AB] localization task and $A B$ recognition task). In CFS, participants localized a face presented in one of four locations as quickly as possible. In the AB experiments, faces (T2) were presented with a short (200 ms) or long (800 ms) lag after a (green) T1 stimulus that participants were asked to identify. In the AB-localization task, participants then indicated T2 location. In the AB-recognition task participants indicated whether T2 was previously presented in the value-learning task ("old") or not ("new"). 


\section{Experiment 1: Continuous flash suppression (CFS)}

To determine whether acquired affective and motivational value can influence awareness of faces, we measured suppression times with a breaking CFS paradigm after faces had been paired with different probabilities of monetary gain and loss in a choice game. If motivational relevance boosted visual awareness, we would expect shorter suppression times for high-compared to low-probability faces, independent of affective valence (win or loss). If reward signals had attentional priority (Raymond \& O’Brien, 2009), the effect could be expected to be larger for win-associated faces. Alternatively, if visual stimuli with negative valence were prioritized for awareness (Gayet et al., 2016; Yang et al., 2007), we would expect shorter suppression times for loss-associated faces.

\section{Method}

Participants. Fifty-eight undergraduate students were recruited through the Oberlin College participant pool. In both this and the subsequent experiments, we only tested participants who said they had normal or corrected-to-normal vision. In addition, all participants were naïve to the research question, and received either course credit or a small monetary compensation for their participation. Informed consent for Experiment 1 was obtained following a protocol approved by the Oberlin College Institutional Review board. One participant was excluded because localization accuracy was only $56 \%$ correct; four other participants needed to be excluded because their data were not stored. The final sample consisted of 53 participants (41 female, mean age 19.3 years, $S D=1.4$ ). Participants received written and verbal instructions and practice trials, and all experiments contained several short obligatory breaks.

Sample size and statistical power. It was not clear which specific effect size reported in previous studies to base the power calculation on. In their AB-recognition study, Raymond and O'Brien (2009) report as the key effect a significant two-way interaction between lag and valence (indicating a reduced $\mathrm{AB}$ for win-associated faces) with an effect size $d_{z}$ of 0.88 . To achieve $80 \%$ power to detect this effect size, only 13 participants would have been required. We decided to test a much larger sample size to increase our chances of detecting a possible effect in the CFS

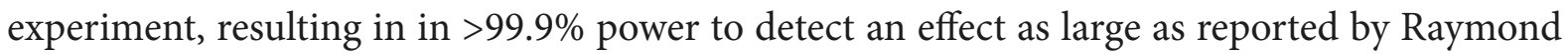
and O'Brien, and in $80 \%$ power to detect effects $d_{z}>0.40$. In Experiments $2 \mathrm{a}$ and $2 \mathrm{~b}$ we also had $>99.9 \%$ power to detect an effect as large as reported by Raymond and O’Brien, and $80 \%$ power to detect effects $d_{z}>0.42$, respectively. 
Display and apparatus. Stimuli were presented on a 24-inch LCD monitor $(1920 \times 1080$ pixels resolution, $60 \mathrm{~Hz}$ refresh rate) that participants viewed dichoptically through a custombuilt mirror stereoscope using a chin-and-head rest placed approximately $84 \mathrm{~cm}$ away from the screen. The mirrors of the stereoscope were adjusted for each observer to yield stable binocular fusion. Experiments were programmed in Matlab using Psychtoolbox(Brainard, 1997) functions. Presentation times were synchronized with the vertical refresh cycle of the screen. Two fusion contours $\left(4.8^{\circ} \times 4.8^{\circ}\right.$ of visual angle $)$ consisting of random black and white pixels (width $0.2^{\circ}$ ) were displayed side-by-side on the screen such that one contour was shown to each eye (distance between the centers of the two contours $10^{\circ}$ ). A small black fixation cross was presented in the center of each contour, and the remainder of the space enclosed by the contour was mid-gray. Participants were asked to maintain fixation throughout the CFS experiment (moving the eyes between trials if necessary).

Stimuli. Stimuli were photographs of 16 faces (eight female) with emotionally neutral expression from the FACES database (Ebner, Riediger, \& Lindenberger, 2010). Photographs were converted to grayscale and cropped to an oval $\left(1.2^{\circ} \times 1.8^{\circ}\right)$, removing hair and outer facial features. Pixel values within the oval were equated for mean and standard deviation. To induce CFS, we generated 170 masks $\left(4.8^{\circ} \times 4.8^{\circ}\right)$ consisting of randomly arranged circles of different sizes in black, white, and various shades of gray (diameter $0.1-0.8^{\circ}$ ).

Value-learning task. Experimental sessions began with a choice game, in which participants could win a small amount of extra money (see Figure 1a). On every trial, participants selected one face from a pair of faces presented simultaneously to both eyes either left and right of fixation (half the trials, centered at an eccentricity of $1.4^{\circ}$ ) or above and below fixation (half the trials, centered at an eccentricity of $1.1^{\circ}$ ). There were two "win" pairs, two "loss" pairs, and two "neutral" pairs. For each type of pair, one of the two pairs consisted of two male faces, and the other pair of two female faces. On win and loss trials participants could win or lose five cents, or gain nothing. For win pairs, the probability of winning five cents was $80 \%$ for one face exemplar (high motivational salience), and $20 \%$ for the other face exemplar (low motivational salience). For loss pairs, the probability of losing five cents was $80 \%$ for one face exemplar (high motivational salience), and $20 \%$ for the other face exemplar (low motivational salience). Neutral pairs never resulted in monetary gains or losses. The three types of pairs (win, loss, neutral) yielded five conditions differing in expected value $(-0.8,-0.2,0,0.2,0.8)$. The assignment of the 16 face exemplars to the pairs was counterbalanced between participants. 
Four exemplars were not shown in the value-learning task and served as "novel" faces in the CFS task (see below). Each trial began with a 1-s blank screen with the contours only, and a 1s fixation period, followed by a pair of faces. Location of the face exemplars was randomized from trial to trial. Participants selected one of the two faces using the arrow keys, with no speed pressure. Upon selection, they received feedback ("WIN" in green font, "LOSS" in red font, or "NOTHING" in black font), together with a summary of their total monetary gains up to this trial. The feedback remained on the screen for $1.3 \mathrm{~s}$. Participants were instructed to maximize their payoff. There were 600 trials, in which each combination of three pair types (rewarded, punished, neutral), two face pairs (male, female), and two stimulus locations (vertical vs. horizontal axis) occurred equally often. Trial order was randomized.

CFS task. A few minutes after completion of the value-learning task, participants completed the CFS task, where we recorded suppression times for the faces now differing in learned expected value, plus four "novel" face exemplars that had not been used in the valuelearning task (Figure 1b). Each trial started with a 1-s fixation period in which only the fusion contours and the fixation crosses were presented; the fixation crosses then turned off for 750 $\mathrm{ms}$, and turned on again for $200 \mathrm{~ms}$ to mark the beginning of a trial. CFS masks changing every $100 \mathrm{~ms}$ were then presented to one eye, and a face was gradually introduced to the other eye by decreasing its transparency to zero over the first second of a trial. Beginning one second after trial onset, the contrast of the CFS masks was linearly decreased to zero over $10 \mathrm{~s}$ in order to force eventual breakthrough. The face was presented until response, or for a maximum trial length of $12 \mathrm{~s}$. Faces were presented in one of the four locations from the value-learning task (either above, below, left or right of fixation, selected at random for every trial). Participants were asked to press one of the four arrow keys on the keyboard corresponding to the four possible face locations to indicate as quickly and accurately as possible in which location a face or any part of a face became visible. For 38 participants the CFS task consisted of 256 trials, in which all combinations of two eyes for face presentation and 16 face exemplars occurred 16 times. Trial order was randomized. Fifteen participants completed 320 trials, in which we randomly intermixed 64 trials with inverted versions of the face exemplars (rotated by 180 degrees, i.e. each of the 16 face exemplars shown two times to each eye, at a random location), and participants were informed about this additional manipulation. We included inverted faces to ensure that our CFS setup would be sufficiently sensitive to detect the well-established faceinversion effect. 
Analyses. For the value-learning task, the probability of optimal choice was calculated for ten trial bins containing 60 trials each. For win pairs, the optimal choice was the $80 \%$-win face. For loss pairs, the optimal choice was the 20\%-loss face. For the neutral condition, choice probability of one arbitrarily selected face is plotted for comparison (Figures 2a, 3a, and 4a). Because proportional data such as choice rates or accuracy are not strictly normally distributed, descriptive statistics in the text are given as median and interquartile range, and all statistical analyses were carried out following a rationalized arcsine transformation (RAU, where a score of 50 corresponds to $50 \%$ correct, with RAU values scaling between -23 and 123). For the CFS task trials with incorrect or no localization responses $(M d n=1.2 \%, I Q R=1.3)$ were excluded from all analyses. As suppression times also violate the assumption of normality they were $\log _{10^{-}}$ transformed (Gayet \& Stein, 2017) before condition means were calculated and statistical analyses were carried out. For illustration purposes and easy eyeballing of the results in standard units $\log _{10}$-transformed suppression times were transformed back (see descriptive statistics and Figure $2 b$; for $\log _{10}$-transfomred suppression times see Figure 3).

Statistics. We report both standard frequentist statistics and Bayes factors (BFs) calculated in JASP (JASP Team, 2020) with default prior scales (Cauchy distribution, scale 0.707). When frequentist statistics indicate a significant effect, the corresponding BF quantifies the evidence for the alternative hypothesis $\left(\mathrm{BF}_{10}\right)$; when the effect is not significant, the reported $\mathrm{BF}$ quantifies the evidence for the null hypothesis $\left(\mathrm{BF}_{01}\right)$. For multi-factorial ANOVAs, we report the inclusion BF quantifying the evidence for all models containing a particular effect compared to all models without that effect. When the assumption of sphericity was violated, we report degrees of freedom and $p$-values after Greenhouse-Geisser correction. 
a

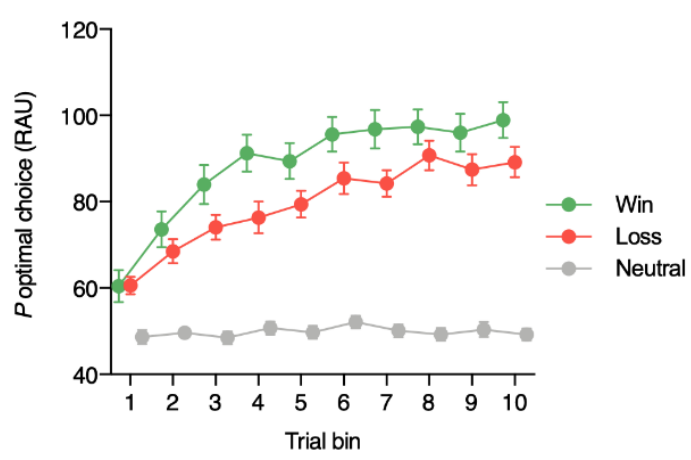

b

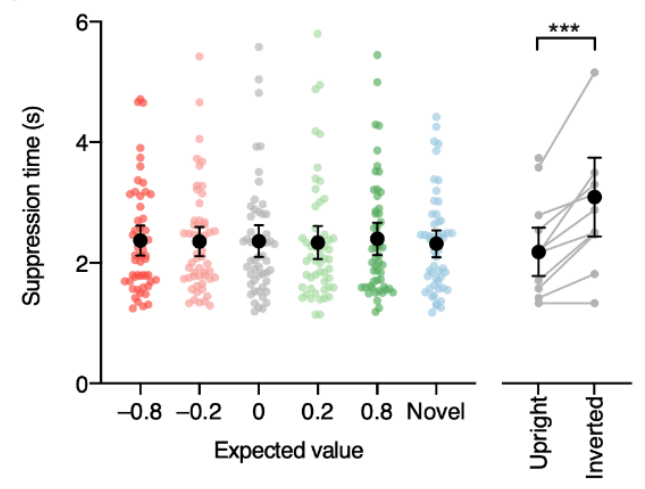

Figure 2. Results from Experiment 1 (CFS). (a) Mean probability of optimal choice in the valuelearning task for ten trial bins of 60 trials each. Error bars represent between-subjects SEs. Note that all proportions (optimal choice, accuracy, hit rates) are plotted as rational arcsine (RAU) scores (where a score of 50 corresponds to a proportion of 0.5 , with RAU values scaling between -23 and 123). (b) Mean suppression times in the breaking CFS task for the five conditions with different expected outcome $(-0.8,-0.2,0,0.2,0.8)$ and for "novel" faces that were not been shown in the valuelearning task. Also shown is the face-inversion effect (difference between upright and inverted faces, $\left.{ }^{* * *} p<.001\right)$ that was tested in a subset of fifteen participants. For Figure $2 b, \log _{10}$-transformed suppression times were transformed back to standard units. Every colored circle represents a participant $(N=53)$; black circles represent the mean and error bars the $95 \% \mathrm{Cls}$.

\section{Results and Discussion}

Value-learning task. As can be seen in Figure 2a, across win and loss pairs probability of optimal choice increased over the ten trial bins in a session $(F(3.99,207.39)=35.50, p<.001$, $\left.\eta_{p}{ }^{2}=.41, \mathrm{BF}_{10}=1.05 \times 10^{46}\right)$. Choices were better for win than for loss pairs $(F(1,52)=10.89, p$ $\left.=.002, \eta_{p}^{2}=.17, \mathrm{BF}_{10}=2.28 \times 10^{19}\right)$. The interaction between valence and trial bin was not significant $\left(F(4.92,255.82)=1.64, p=.15, \eta_{p}{ }^{2}=.03, \mathrm{BF}_{01}=86.19\right)$. Performance appeared to have reached asymptote after six trials bins (of 60 trials each) for both win pairs ( $M d n=94.7 \%$ correct, $I Q R=24.8)$ and loss pairs $(M d n=86.4 \%$ correct, $S D=24.7)$. For both win- and loss pairs, in the sixth bin performance was significantly better than in the previous bin $(p<.05)$ but did not differ significantly from the following bins $(p>.10)$ Overall choice performance was similar to previous studies using comparable value-learning tasks with faces.

CFS task. In brief, suppression times were similar for faces with different expected values, and statistical analyses did not reveal any indication of effects of valence (rewarded, punished) or motivational salience $(80 \%$ vs. $20 \%$ outcome probability; see Figure 2$)$. An 
ANOVA with the factors valence and motivational salience revealed no significant effects (valence, $F(1,52)=0.42, p=.52, \eta_{p}^{2}<.01, \mathrm{BF}_{01}=5.99$; motivational salience, $F(1,52)=0.62, p$ $=.43, \eta_{p}^{2}=.01, \mathrm{BF}_{01}=4.11$; interaction, $\left.F(1,51)=0.91, p=.34, \eta_{p}^{2}=.02, \mathrm{BF}_{01}=3.55\right)$. We carried out a set of 15 paired sample $t$-tests comparing all conditions (uncorrected for multiple comparisons) to ensure that we did not miss a potential effect. However, there were no significant differences between any of the conditions, including the comparisons with novel faces (all $\left.t(52)<1.27, p>.21, d_{z}<0.18, \mathrm{BF}_{01}>3.15\right)$. Thus, there was no effect of value learning on awareness of faces in CFS. To our surprise these data also do not replicate our previous finding of overall shorter suppression times for learned vs. novel faces.

To check whether our CFS task had enough sensitivity to detect possible differences in suppression times, we measured the well-established face-inversion effect (Jiang et al., 2007; Stein, 2019; Stein, Sterzer, \& Peelen, 2012) in a subset of fifteen participants. As is commonly found, suppression times for upright faces $(M=2.29 \mathrm{~s})$ were (much) shorter than for inverted faces $(M=3.24 \mathrm{~s})$, with substantial evidence for an effect of inversion $\left(t(14)=7.26, p<.001, d_{z}\right.$ $\left.=1.87, \mathrm{BF}_{10}=4.84 \times 10^{3}\right)$. This demonstrates that our CFS setup was capable of detecting effects.

Finally, we compared suppression times between the first and the second half of the CFS task. This was done to check for potentially short-lived learning effects (Rothkirch et al., 2013), and to test whether overall suppression weakened over time, as is commonly found (Ludwig, Sterzer, Kathmann, Franz, \& Hesselmann, 2013; Stein, Siebold, \& Van Zoest, 2016). As can be seen in Figure 3, while there was no evidence for effects of value learning on suppression times in either the first or in the second half of the experiment, in all conditions suppression times became shorter in the second half of the experiment (paired sample $t$-tests, all $t(52)<4.17, p<$ $\left..001, d_{z}>0.57, \mathrm{BF}_{01}>197.37\right)$. Thus, suppression times for faces showed the established patterns of being shorter for upright than inverted faces and of becoming shorter over time, but were not influenced by value learning. 


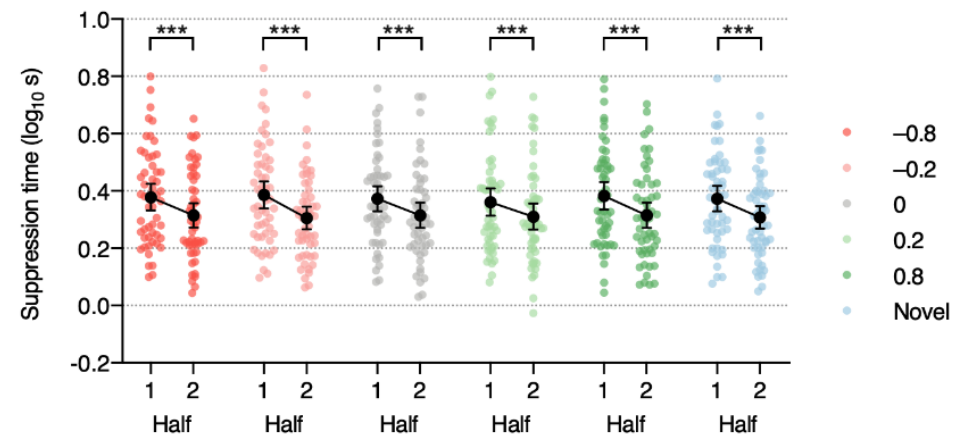

Figure 3. Results from Experiment 1 (CFS), shown separately for the first and the second half of the experiment. Different colors represent different conditions differing in expected value, plus the novel-face condition. Every colored circle represents a participant $(N=53)$; black circles represent the mean and error bars the $95 \% \mathrm{Cls}$. ${ }^{* *} p<.001$.

\section{Experiment 2: Attentional blink (AB)}

To determine whether the absence of effects in CFS reflected the perceptual nature of our task (simple localization) or properties of the method, such as CFS abolishing processing early in the visual system (Moors, Hesselmann, Wagemans, \& van Ee, 2017), we conducted two attentional-blink experiments $(\mathrm{AB})$. In models of consciousness, such as global neuronal workspace theory (Dehaene, Changeux, Naccache, Sackur, \& Sergent, 2006), a distinction has been made between visual processing of stimuli with reduced stimulus strength (such as in CFS) vs. stimuli with greater stimulus strength under limited attention (such as in the AB), with more extensive processing taking place in the latter case. In Experiment 2a (AB-localization), participants localized a face that followed an attention-demanding first target with a short or long lag. Similar to the CFS experiment, the localization task did not require memory retrieval. If acquired value influenced basic face perception and awareness, we would expect an effect on overall localization performance. If acquired value influenced attention, we would expect differential effects for the two lags (i.e. influence on $A B$ magnitude), with a reduced or eliminated $\mathrm{AB}$ for win-associated faces (Raymond \& O’Brien, 2009). Experiment 2b was a conceptual replication of Raymond and O'Brien's study, where participants indicated whether shown in an $\mathrm{AB}$ sequence had previously been presented in the value-learning task. If acquired value modulated memory for faces, we would expect an effect on overall recognition accuracy. If acquired value also influenced attention in this task, as reported by Raymond and O'Brien we would expect an effect on $\mathrm{AB}$ magnitude, with a smaller $\mathrm{AB}$ for win-associated faces. 


\section{Method}

Participants. One hundred participants, most of them undergraduate psychology students, were recruited through the University Amsterdam participant pool. Informed consent was obtained following a protocol approved by the University of Amsterdam local ethics committee. Half of the participants were assigned to the AB-localization task (Experiment 2a), and half to the AB-recognition task (Experiment $2 \mathrm{~b}$ ). A total of four of the 100 participants were excluded due to poor overall performance, indicating failure to follow instructions. In the AB-localization experiment one participant whose overall localization accuracy was below the chance level of $50 \%$ was excluded. In the AB-recognition experiment three participants whose overall recognition sensitivity was below the chance level of $d^{\prime}=0$ were excluded. The final samples consisted of 49 participants in the AB-localization experiment ( 2 female, mean age 24.3 years, $S D=8.4$ ), and 47 participants in the $A B$-recognition experiment (31 female, mean age 23.0 years, $S D=6.4)$.

Display, apparatus, and stimuli. The overall setup was similar to Experiment 1, except that participants viewed the screen binocularly from a free viewing distance of approximately $70 \mathrm{~cm}$, and the contour in which stimuli were presented was displayed in the center of the screen (Figure 1b). As we used stimuli of identical pixel dimensions in all experiments, depending on participants' free viewing distance the retinal projections of the stimuli in the $\mathrm{AB}$ experiment were slightly larger than in Experiment 1. Stimuli were the same photographs as in Experiment 1. For the AB-recognition task we added six faces that served as additional "novel" stimuli, such that for every participant there were 12 "old" face exemplars seen in the value-learning task, and 10 "novel" face exemplars. The assignment of face exemplars was counterbalanced between participants. For the AB experiments 154 scrambled distracters were generated by dividing the face stimulus ovals into grids consisting of 16 or 20 rectangles of different sizes and randomly rearranging these rectangles. As a first attention-demanding target (T1), we created 88 stimuli that consisted either of a random arrangement of differently sized circles or rectangles. These were then cut to correspond to the face ovals and displayed through the green RGB channel only (rendering T1 salient in the context of gray faces and distracters).

Value-learning task. The value-learning task was similar to Experiment 1, with the following exceptions. Face pairs were shown only in the left or right location (corresponding to the two locations in the AB-localization task), and we reduced the number of trials to 360, as 
Experiment 1 had indicated that performance reached asymptote after six trial bins of 60 trials each.

AB-localization task. Each trial started with 1-s fixation, which then turned off for 500 ms to mark the beginning of the RSVP sequence. Three different streams of 24 randomly selected scrambled distracters changing every $100 \mathrm{~ms}$ were presented in the left, right, and central location within the contour (Figure 1b). After the presentation of seven to eleven distracters (selected at random), T1 (green circles or diamonds) was presented in the central stream; the face stimulus followed after one or seven distracters in the left or right stream (resulting in T1-T2 SOAs of 200 and $800 \mathrm{~ms}$, corresponding to "lag 2" and "lag 8" conditions). At the end of the stimulus presentation sequence, participants first indicated T1 identity using the " 1 " (circles) and " 2 " (rectangles) keys, and then indicated face location using the left and right arrow keys. In both $\mathrm{AB}$-localization and $\mathrm{AB}$-recognition, instructions emphasized the importance of getting the T1 identity right, and participants were instructed to respond as accurately as possible, without speed pressure. The AB-localization task consisted of 384 trials, in which all combinations of two lags, two face locations, two T1 identities, and 16 face exemplars occurred six times. Trial order was randomized.

AB-recognition task. Each trial started with 1-s fixation, which then turned off for 500 ms to mark the beginning of the stimulus presentation sequence. All stimuli were presented in the center of the central contour (Figure 1c). T1 was presented for $100 \mathrm{~ms}$, followed by a scrambled distracter for $100 \mathrm{~ms}$, and a blank contour for $100 \mathrm{~ms}$ ("lag 2") or for $700 \mathrm{~ms}$ ("lag 8"), followed by face stimulus for $100 \mathrm{~ms}$, and another scrambled distracter for $100 \mathrm{~ms}$. Participants then indicated T1 identity using the "1" (circles) and "2" (rectangles) keys, and then whether the face was "old" (presented in the preceding value-learning task) or "new" (not presented in the value-learning task) using the left and right arrow keys. There were 440 trials, in which all combinations of two lags, two T1 identities, and 22 face exemplars occurred five times. Trial order was randomized.

Analyses. For the value-learning task, analyses were the same as in Experiment 1. For the $\mathrm{AB}$-tasks only trials with correct $\mathrm{T} 1$ identification were included in the analyses (ABlocalization task, $M d n=95.1 \%$ correct, $I Q R$ 4.0; AB-recognition task, $M d n=88.2 \%$ correct, $I Q R$ 10.0). All statistical analyses of proportion of correct responses were carried out on RAU scores, while descriptive statistics in the text refer to the non-transformed proportions. In the $\mathrm{AB}$-localization task, accuracy represents a criterion-free estimate of localization performance 
(chance level $50 \%$ correct). The AB-recognition task, in contrast, is a "yes/no" task, and we report proportion correct for the different conditions (for old faces, this corresponds to the hit rate; for new faces, this corresponds to the correct-rejection rate). Although a previous study reported d' for old faces in this recognition task (Raymond \& O'Brien, 2009), d' for the different conditions would be based on identical false-alarm rates (new faces did not differ with regard to expected value) and thus differences between value conditions can, by definition, only influence the hit rate, so that $d$ ' for the different conditions would not represent a criterion-free index of sensitivity but would be equivalent to the hit rates reported here.

a

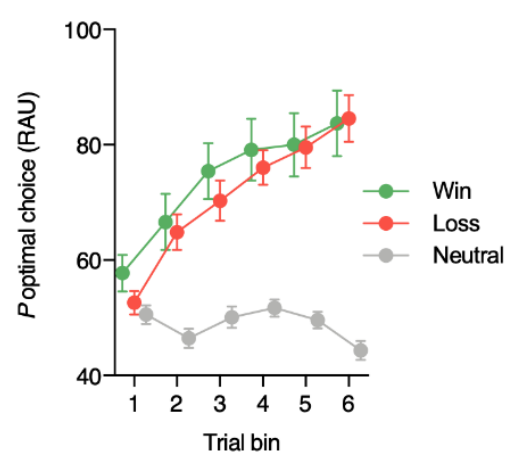

b

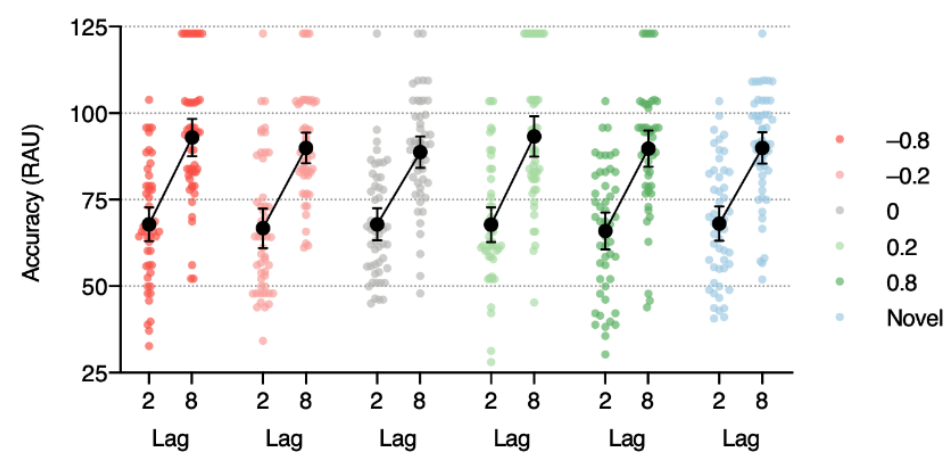

Figure 4. Results from Experiment 2a (AB-localization). (a) Mean probability of optimal choice in the value-learning task for six trial bins of 60 trials each. Error bars represent between-subjects SEs. (b) Face localization accuracy in the AB-localization task, shown separately for lag 2 and lag 8 , for the five conditions with different expected outcome $(-0.8,-0.2,0,0.2,0.8)$ and for "novel" faces that were not shown in the value-learning task. Every colored circle represents a participant $(N=49)$; black circles represent the mean and error bars the $95 \% \mathrm{Cls}$. 


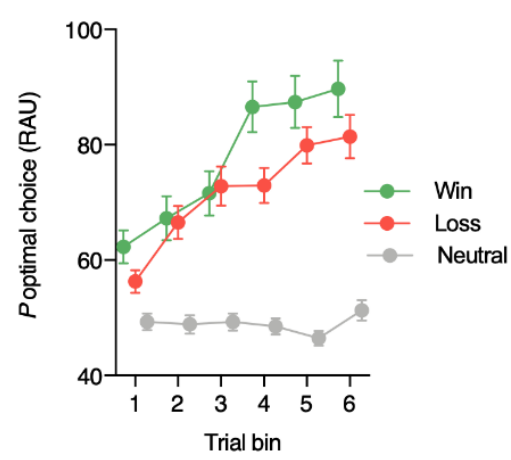

b

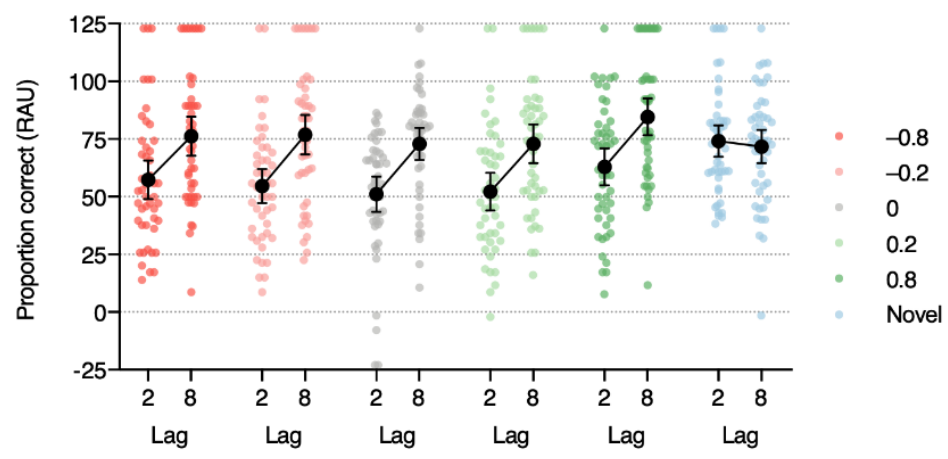

Figure 5. Results from Experiment 2b (AB-recognition). (a) Mean probability of optimal choice in the value-learning task for six trial bins of 60 trials each. Error bars represent between-subjects SEs. (b) Proportion of correct responses in the AB-recognition task, shown separately for lag 2 and lag 8, and for the five conditions with different expected outcome $(-0.8,-0.2,0,0.2,0.8)$. For these "old" faces that were shown in the value-learning task proportion correct reflects the hit rate. For "novel" faces that were not shown in the value-learning task proportion correct reflects the correct rejection rate. Every colored circle represents a participant $(N=47)$; black circles represent the mean and error bars the $95 \% \mathrm{Cls}$.

\section{Results and Discussion}

Value-learning task. As can be seen in Figure $4 \mathrm{a}(\mathrm{AB}-$ localization) and Figure $5 \mathrm{a}(\mathrm{AB}-$ recognition), value learning was similar for the two experiments, and we collapsed the data from the 96 participants for the following analyses. Across win and loss pairs probability of optimal choice increased over the six trial bins in a session $(F(3.04,288.93)=58.89, p<.001$, $\left.\eta_{p}{ }^{2}=.38, \mathrm{BF}_{10}=4.45 \times 10^{35}\right)$, and choices were better for win than for loss pairs $(F(1,95)=6.14$, $\left.p=.015, \eta_{p}^{2}=.06, \mathrm{BF}_{10}=3.21 \times 10^{4}\right)$. The interaction between valence and trial bin was not significant $\left(F(3.42,324.69)=0.85, p=.48, \eta_{p}{ }^{2}<.01, \mathrm{BF}_{01}=241.04\right)$. Overall performance after six trial bins for win pairs $(M d n=91.6 \%$ correct, $I Q R=45.1)$ and loss pairs $(M d n=81.8 \%$ correct, $I Q R=30.3$ ) was similar to Experiment 1 (independent samples $t$-tests, both $t(147)<$ $\left.1.54, p>.12, \mathrm{BF}_{01}>1.86\right)$, and there was substantial variation between participants.

AB-localization task. Figure $4 \mathrm{~b}$ shows face-localization accuracy for the different conditions and lags. A three-way repeated measures ANOVA including lag (2 vs. 8) and the four key conditions that differed in valence and motivational salience (win vs. loss, $80 \%$ vs. $20 \%$ outcome probability) revealed a significant main effect of lag $\left(F(1,48)=131.80, p<.001, \eta_{p}^{2}=\right.$ $\left..73, \mathrm{BF}_{10}=1.14 \times 10^{51}\right)$, with better performance at lag $8(M d n=88.1 \%$ correct, $I Q R=12.8)$ than 
at lag $2(M d n=64.2 \%$ correct, $I Q R=21.4)$, and a two-way interaction between valence and motivational salience $\left(F(1,48)=5.19, p=.027, \eta_{p}{ }^{2}=.10\right.$, but $\left.\mathrm{BF}_{10}=1.02\right)$, but no other significant effects (all $F<0.75, p>.39, \eta_{p}^{2}<.02, \mathrm{BF}_{01}>3.67$ ). The two-way interaction reflected an unexpected pattern of results. For loss-pairs, performance tended to be better for highprobability faces than for low-probability faces, whereas for win-pairs performance tended to be better for low-probability faces than for high-probability faces (Figure $4 \mathrm{~b}$ ). Considering that the Bayes factor for this interaction indicates that the null hypothesis is equally likely as the alternative hypothesis, we did not further explore this effect. A set of six paired sample $t$-tests comparing localization performance between the long and the short lag for every condition (uncorrected for multiple comparisons) revealed significantly better performance at lag 8 than at lag 2 for all conditions (all $t(45)>7.66, p<.001, d_{z}>1.09, \mathrm{BF}_{10}>1.51 \times 10^{7}$ ). However, $\mathrm{AB}$ magnitude did not differ significantly between conditions (as indicated by the absence of significant interactions with lag). Together, these results provide no evidence for a reduced $\mathrm{AB}$ for win-associated faces, or for better localization of faces with high motivational salience.

AB-recognition task. Analogous analyses carried out on the hit rates for the four key conditions in the $\mathrm{AB}$-recognition task (see Figure $5 \mathrm{~b}$ ) revealed a significant main effect of lag $\left(F(1,46)=95.02, p<.001, \eta_{p}^{2}=.67, \mathrm{BF}_{10}=1.04 \times 10^{17}\right)$, with higher hit rates at lag $8(M d n=$ $73.1 \%, I Q R=25.6)$ than at lag $2(M d n=55.4 \%, I Q R=33.4)$, a significant effect of motivational salience $\left(F(1,46)=6.46, p=.014, \eta_{p}{ }^{2}=.12, \mathrm{BF}_{10}=6.40\right)$, with higher hit rates for faces leading to an outcome with $80 \%$ probability $(M d n=69.6 \%, I Q R=31.4)$ than for faces leading to an outcome with only $20 \%$ probability $(M d n=62.6 \%, I Q R=25.9)$, and a significant interaction between valence and motivational salience $\left(F(1,46)=5.22, p=.027, \eta_{p}{ }^{2}=.10\right.$, but $\left.\mathrm{BF}_{10}=2.29\right)$, but no other significant effects (all $F<0.71, p>.40, \eta_{p}^{2}<.02, \mathrm{BF}_{01}>4.32$ ). Although Bayes factor revealed only anecdotal evidence for the valence-by-salience interaction, from Figure $5 b$ it is clear that the effect reflected particularly high hit rates for high-probability win-associated faces. For loss-associated faces, there was no significant difference between high- and low-probability faces $\left(t(46)=0.22, p=.83, d_{z}=0.03, \mathrm{BF}_{01}=6.18\right)$. For win-associated faces, by contrast, hit rates were significantly higher for high-probability faces than for low-probability faces $(t(46)=3.80$, $\left.p<.001, d_{z}=0.55, \mathrm{BF}_{10}=63.36\right)$. Hit rates for high-probability win-associated faces were also significantly higher than for neutral and low-probability loss-associated faces (both $t(46)>3.48$, $\left.p<.002, d_{z}>0.42, \mathrm{BF}_{10}>5.84\right)$, and tended to be somewhat higher than for high-probability loss-associated faces $\left(t(46)=2.30, p=.026, d_{z}=0.34\right.$, but $\left.\mathrm{BF}_{10}=1.70\right)$. These results indicate 
better recognition memory for faces with high motivational salience, and this effect was particularly pronounced for win-associated faces.

Finally, paired sample $t$-tests revealed a significant AB (higher hit rates at lag 8 than at lag 2) for all five "old" conditions (faces presented in the value-learning task, all $t(46)>5.57, p$ $<.001, d_{z}>0.81, \mathrm{BF}_{10}>1.34 \times 10^{4}$ ). For "new" faces (faces not presented in the value-learning task) correct rejection rates did not differ significantly between lag 8 and lag $2(t(46)=0.78, p$ $\left.=.44, d_{z}=0.11, \mathrm{BF}_{01}=4.47\right)$. Overall mean recognition $d^{\prime}$ was $0.87(S D 0.68)$ at lag 2 and 1.35 ( $S D$ 0.81) at lag 8, similar to the AB-recognition study by Raymond and O'Brien (2009), but AB magnitude did not differ between conditions (as indicated by the absence of significant interactions with lag). In summary, the AB-recognition experiment revealed overall better recognition memory for high-probability win-associated faces but no evidence for a modulation of the $\mathrm{AB}$ by valence or motivational salience.

\section{Overview of key CFS and AB effects}

To facilitate interpretation, we calculated difference scores reflecting the key effects of motivational salience and affective value from the CFS and AB data. The salience effect was calculated as the difference between low- and high-probability faces, the gain effect as the difference between win-associated and neutral faces, and the loss effect as the difference between loss-associated and neutral faces. We also calculated a high-gain and a high-loss effect as the difference between win-/loss associated faces with high outcome probability and neutral faces. Figure 6 shows these key effects, plotted such that positive values indicate an effect in the predicted direction (e.g. a positive gain effect in the CFS experiment would reflect shorter suppression times for win-associated faces than for neutral faces, and higher accuracy/hit rates for win-associated faces than for neutral faces in the $\mathrm{AB}$ experiments). For the $\mathrm{AB}$ experiments, we calculated both overall effects across lag 2 and lag 8 , and blink effects for which we contrasted the $\mathrm{AB}$ size (lag 8 minus lag 2) between conditions (e.g. a positive gain blink effect would reflect a smaller $\mathrm{AB}$ for win-associated faces than for neutral faces).

To test for effects in the predicted direction, all effects were tested against zero with onesided $t$-tests. For CFS, none of the effects were significant, with strong evidence for the null hypothesis (all $t(52)<0.13, p>.45, d_{z}<0.02, \mathrm{BF}_{01}>6.04$ ). For overall accuracy in the ABlocalization experiment, there were no significant effects either, but the data were more variable, resulting in weaker support for the null hypothesis (all $t(48)<1.09, p>.14, d_{z}<0.16, \mathrm{BF}_{01}>$ 
2.17). For overall hit rates in the AB-recognition experiment there were significant effects of salience $\left(t(46)=2.88, p=.003, d_{z}=0.42, \mathrm{BF}_{10}=11.81\right)$, gain $\left(t(46)=1.90, p=.032, d_{z}=0.28\right.$, but $\left.\mathrm{BF}_{10}=1.59\right)$, and high-gain $\left(t(46)=3.91, p<.001, d_{z}=0.57, \mathrm{BF}_{10}=170.16\right)$, but no other significant effects (both $t(46)<1.13, p>.13, d_{z}<0.17, \mathrm{BF}_{01}>2.02$ ). These results confirm our previous analyses: While there was no effect of value learning on suppression times and face localization, recognition performance was better for faces with high motivational salience, and in particular for high-probability win-associated faces. In the $\mathrm{AB}$ experiments, there was no evidence that the magnitude of the blink was influenced by value learning (all $t<0.97, p>.16$, $\left.d_{z}<0.15, \mathrm{BF}_{01}>2.45\right)$. Thus, in the $\mathrm{AB}-$ recognition experiment motivational salience and reward boosted overall recognition but there was no evidence for greater attentional priority of motivationally salient or rewarded faces.

Finally, in all experiments, there was substantial variability in value learning. One concern is that only particularly good learners might have shown an effect. To address this possibility, we considered correlating performance in the value-learning task with the CFS and $\mathrm{AB}$ effects. However, for many participants value-learning was at ceiling (in particular for winassociated face pairs). We therefore calculated all key effects separately for those participants who demonstrated learning defined as performing above a certain criterion, following Rutherford and colleagues (2010). An overview of all effects and individual data points can be found in the Supplementary material (Figure S1). In brief, we obtained a near-identical pattern of results in this subgroup analysis, with no evidence for an effect of value-learning on CFS, ABlocalization, or blink magnitude. 

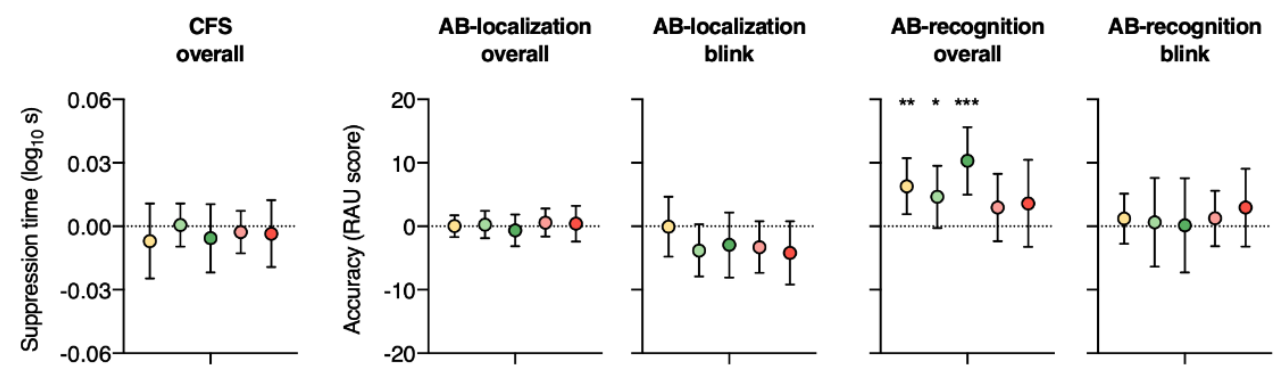

Salience effect

Gain effect

High-gain effect

Loss effect

High-loss effect

Figure 6. Overview of the key effects from CFS, AB-localization, and AB-recognition. Shown are difference scores for overall suppression times (CFS), accuracy (AB-localization), and hit rates (ABrecognition). See text for calculation of effects. Effects are plotted such that positive values indicate an effect in the predicted direction (e.g. a positive gain effect in the CFS experiment would reflect shorter suppression times for win-associated faces than for neutral faces, and higher accuracy/hit rates in the $A B$ experiments). For the $A B$-experiments, also blink effects are shown, where positive values would reflect a reduced $A B$ magnitude in the predicted direction. Circles show means, error bars show $95 \% \mathrm{Cls}$. Stars indicate significant one-sided t-tests testing for an above-zero effect ${ }^{* *} p$ $<.001 .{ }^{* *} p<.01 .{ }^{* * *} p<.05$.

\section{General Discussion}

Although it is widely believed that emotional and motivational value can influence perception of social signals such as faces, there is little unequivocal empirical support for this idea. For example, a recent meta-analysis compared the effects of different emotional stimuli and found that only fearful faces elicited reliable effects on visual perception and attention across a range of different experimental paradigms, such as breaking CFS, binocular rivalry and attentional capture (Hedger et al., 2016). However, fearful faces differ from non-emotional stimuli in terms of such as local contrast (Yang et al., 2007) and effective contrast (Hedger et al., 2015), such that effects could reflect differences in low-level properties rather than genuine influences of emotion on perception. To rule out such low-level explanations, here we associated faces with different affective and motivational value and measured effects on visual awareness, attention, and memory. Following the value-learning procedure, we found that participants were more likely to recognize faces with high motivational salience, but we did not obtain effects on awareness in CFS (Experiment 1), RSVP (Experiment 2a), or attentional priority during the $\mathrm{AB}$ (Experiment $2 \mathrm{a}$ and $2 \mathrm{~b}$ ), suggesting that acquired affective and motivational value affects face perception only at later stages associated with memory. 
In all experiments, results from the value-learning task demonstrated successful learning for both win- and loss-associated faces. Although there was large variability between participants, restricting the analyses to the subset of particularly good learners did not change the pattern of results (Supplementary material). Consistent with previous studies that used affective-learning procedures with faces, in the CFS experiment there was no evidence for effects of value learning on awareness of faces. The strong inversion effect we obtained in a subset of participants demonstrated that our setup was sufficiently sensitive to detect effects. The results from the AB-localization experiment provided further support that basic perception, as measured with localization tasks that were fully orthogonal to the value-learning manipulation, is not influenced by learned emotion and motivation. We recently adopted the same modified RSVP procedure with three parallel streams to measure localization of upright and inverted faces and obtained strong inversion effects similar to CFS with this method (Stein \& Peelen, submitted), indicating that this AB-localization task is, in principle, capable of detecting effects on perception. Note that both the AB-localization and AB-recognition experiments produced strong $\mathrm{AB}$ effects (long vs. short lag), demonstrating that our setup was sensitive to differences in (temporal) attention. Together, the results from the CFS and ABlocalization experiment indicate that for faces acquired value does not influence perception and attention.

Our conclusions are naturally limited to the particular stimuli and value-learning task adopted in our study. As such, they do not contradict effects of emotion on perception obtained with simple stimuli such as colors and gratings following classical fear conditioning procedures. It is possible, for example, that learning signals can modulate neural representations of comparably simple stimuli in early visual cortex (Padmala \& Pessoa, 2008), leading to enhanced perception and attention (Gayet et al., 2016), while they do not affect representations of more complex stimuli such as faces in downstream visual areas representing more complex stimuli such as objects and faces. Similarly, it is possible that the present value-learning task failed to induce sufficiently strong learning signals that would have penetrated visual perception. Pairing stimuli with electrical shocks arguably represents a more powerful manipulation than pairing stimuli with small amounts of monetary gain or loss. However, as we rarely encounter stimuli in our daily lives that are paired with unconditioned stimuli such as electric shocks (or so we hope), our value-learning task with faces may represent a more ecologically valid test of emotional and motivational influences on perception. Together with previous failures to obtain 
effects of affective learning on basic perception of faces, our findings place an upper boundary on the effects of affective learning on perception and attention.

The results from the AB-recognition experiment, which was a conceptual replication of Raymond and O'Brien (2009), came as a surprise. While we replicated overall better recognition of faces that had greater motivational relevance, we did not find a modulation of the $\mathrm{AB}$ or a reduced $\mathrm{AB}$ for win-associated faces. There are several differences between our implementation of the AB-recognition task and the study by Raymond and O'Brien that may account for the different pattern of results. While we used photographs of real faces, Raymond and O'Brien used computer-generated faces. It is possible that in our study participants attended to features of faces (such as the eyebrows) that were informative in the value-learning procedure and a putative enhancement of these features following the choice game did not influence perception or attention in our CFS and $\mathrm{AB}$ tasks. Furthermore, Raymond and O'Brien's participants received additional auditory feedback (beeps) in the choice game, perhaps providing an additional learning signal that was absent in our study. Finally, as performance in the choice game reached asymptote in the CFS experiment after six bins we shortened the value-learning procedure for the $\mathrm{AB}$ experiments, resulting in fewer trials compared to Raymond and O'Brien's study. However, as performance in the value-learning tasks was similar to previous studies, these speculations represent post-hoc explanations for our findings and are not directly supported by data.

In support of a modular view of the mind, Firestone and Scholl (2015) concluded that top-down effects on vision have not been convincingly established and provide a list of six pitfalls that undermine such claims in many studies. The present results, together with previous studies using similar affective-learning procedures with faces, highlight several of their pitfalls. One pitfall pointed out by Firestone and Scholl includes conflating "top-down effects with lowlevel differences", as is common in studies using stimuli with intrinsic emotional value such as fearful faces. Other pitfalls include conflating "perception versus judgment", "perception versus demand and response bias", and "memory and perception" in dependent measures collected in such studies. These issues are reflected in the criterion-sensitive measure of memory adopted in the AB-recognition experiment. Future studies investigating the effect of acquired emotional-motivational value on visual perception and memory need to carefully distinguish between these factors. For now, our results suggest that effects of value learning on face perception are limited to dependent measures that reflect memory and are sensitive to criterion 
effects, and they do not provide support for the idea that motivation and emotion influence basic perception.

\section{Acknowledgments}

We thank Seh Min Suh for help with data collection.

\section{Supplement}

In all experiments, there was substantial variability in value learning. One concern is that only particularly good learners might have shown an effect. To address this possibility, we calculated effects separately for those participants who demonstrated learning defined as performing above a certain (arbitrary) criterion, following Rutherford and colleagues (2010). We set this criterion to choosing the optimal stimulus (high-probability win-face [0.8], lowprobability loss face $[-0.2]$ ) on more than $75 \%$ of the trials in the last bin of the value-learning task. If the respective criterion was achieved for only one of the face pairs, only this pair was included in the analyses. To simplify interpretation, we report the key effects calculated as difference scores for motivational salience and affective value from the CFS and AB data, in a way analogous to the analyses in the main paper (Figure 6). Figure S1 shows all key effects separately for the all participants (same data as in Figure 6) and for those participants who performed above the criterion (>75\% correct). As can be seen in Figure S1, results for these subgroups of particularly good learners were virtually the same as for the whole group, with no evidence for stronger effects of motivation or valence in any of the experiments. 
a

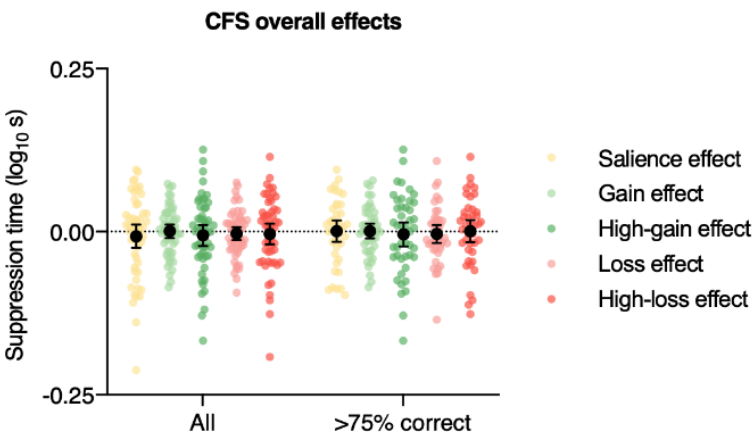

b

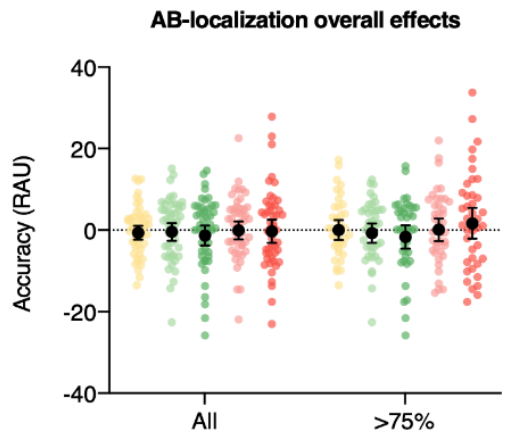

C

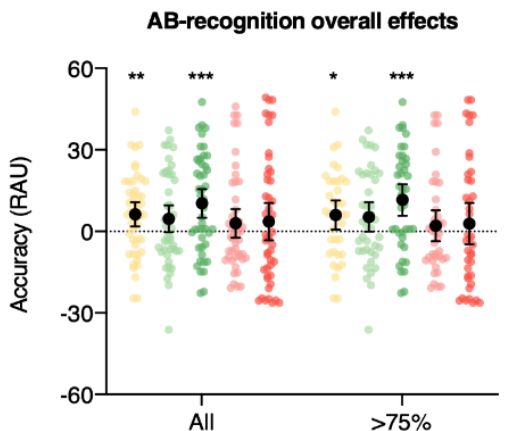

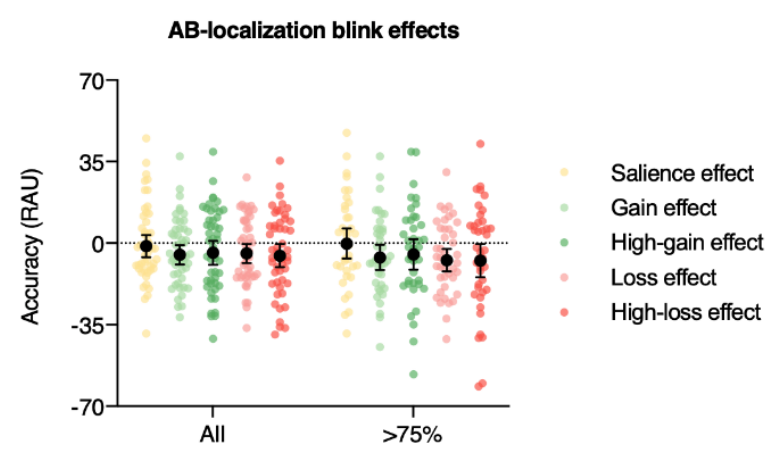

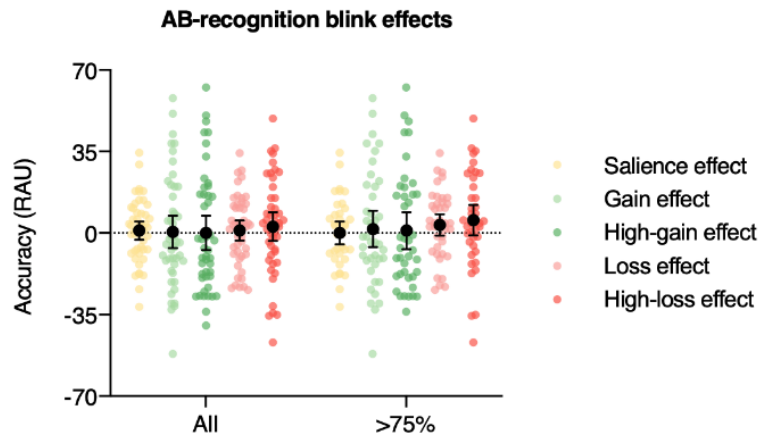

Figure S1. Overview of the key effects from (a) CFS, (b) AB-localization, and (c) AB-recognition. Shown are difference scores for overall suppression times (CFS), accuracy (AB-localization), and hit rates (ABrecognition). See the main text for calculation of effects. Effects are plotted such that positive values indicate an effect in the predicted direction (e.g. a positive gain effect in the CFS experiment would reflect shorter suppression times for win-associated faces than for neutral faces, and higher accuracy/hit rates in the $A B$ experiments). For the $A B$-experiments, also blink effects are shown, where positive values would reflect a reduced $A B$ magnitude in the predicted direction. Every colored circle represents a participant, black circles the means, and error bars show $95 \% \mathrm{Cls}$. Stars indicate significant $t$-tests (here, two-tailed) against chance ${ }^{* *} p<.001 .{ }^{* *} p<.01 .{ }^{* * *} p<.05$. 


\section{References}

Bourgeois, A., Chelazzi, L., \& Vuilleumier, P. (2016). How motivation and reward learning modulate selective attention. Progress in Brain Research, 229, 325-342. https://doi.org/10.1016/bs.pbr.2016.06.004

Brainard, D. H. (1997). The psychophysics toolbox. Spatial Vision, 10, 433-436.

Dehaene, S., Changeux, J. P., Naccache, L., Sackur, J., \& Sergent, C. (2006). Conscious, preconscious, and subliminal processing: A testable taxonomy. Trends in Cognitive Sciences, 10(5), 204-211. https://doi.org/10.1016/j.tics.2006.03.007

Ebner, N. C., Riediger, M., \& Lindenberger, U. (2010). FACES-a database of facial expressions in young, middle-aged, and older women and men: Development and validation. Behavior Research Methods, 42(1), 351-362. https://doi.org/10.3758/BRM.42.1.351

Gayet, S., Stein, T., \& Peelen, M. V. (2019). The danger of interpreting detection differences between image categories: A brief comment on "mind the snake: Fear detection relies on low spatial frequencies" (gomes, soares, silva, \&amp; silva, 2018). Emotion, 19(5). https://doi.org/10.1037/emo0000550

Gayet, S., Paffen, C. L. E., Belopolsky, A. V., Theeuwes, J., \& Van der Stigchel, S. (2016). Visual input signaling threat gains preferential access to awareness in a breaking continuous flash $\begin{array}{lllll}\text { suppression } & \text { paradigm. } & \text { Cognition, } & \text { 77-83, }\end{array}$ https://doi.org/10.1016/j.cognition.2016.01.009

Gayet, S., \& Stein, T. (2017). Between-subject variability in the breaking continuous flash suppression paradigm: Potential causes, consequences, and solutions. Frontiers in Psychology, 8, 437. https://doi.org/10.3389/fpsyg.2017.00437

Gray, K. L. H., Adams, W. J., Hedger, N., Newton, K. E., \& Garner, M. (2013). Faces and awareness: Low-level, not emotional factors determine perceptual dominance. Emotion, 13(3), 537-544. https://doi.org/10.1037/a0031403

Hedger, N., Adams, W. J., \& Garner, M. (2015). Fearful faces have a sensory advantage in the competition for awareness. Journal of Experimental Psychology: Human Perception and Performance, 41(6), 1748-1757.

Hedger, N., Gray, K. L. H., Garner, M., \& Adams, W. J. (2016). Are visual threats prioritized without awareness? A critical review and meta-analysis involving 3 behavioral paradigms and 2696 observers. Psychological Bulletin, 142(9), 934-968. https://doi.org/10.1037/bul0000054 
Jiang, Y., Costello, P., \& He, S. (2007). Processing of invisible stimuli: Advantage of upright faces and recognizable words in overcoming interocular suppression. Psychological Science, 18(4), 349-355. https://doi.org/10.1111/j.1467-9280.2007.01902.x

Ludwig, K., Sterzer, P., Kathmann, N., Franz, V. H., \& Hesselmann, G. (2013). Learning to detect but not to grasp suppressed visual stimuli. Neuropsychologia, 51(13), 2930-2938. https://doi.org/10.1016/j.neuropsychologia.2013.09.035

Moors, P., Gayet, S., Hedger, N., Stein, T., Sterzer, P., van Ee, R., Wagemans, J., \& Hesselmann, G. (2019). Three criteria for evaluating high-level processing in continuous flash suppression. Trends in Cognitive Sciences, 23(4), 267-269. https://doi.org/10.1016/j.tics.2019.01.008

Moors, P., Hesselmann, G., Wagemans, J., \& van Ee, R. (2017). Continuous Flash Suppression: Stimulus Fractionation rather than Integration. Trends in Cognitive Sciences, 21(10), 719721. https://doi.org/10.1016/j.tics.2017.06.005

Padmala, S., \& Pessoa, L. (2008). Affective learning enhances visual detection and responses in primary visual cortex. Journal of Neuroscience, 28(24), 6202-6210. https://doi.org/10.1523/JNEUROSCI.1233-08.2008

Rabovsky, M., Stein, T., \& Abdel Rahman, R. (2016). Access to awareness for faces during continuous flash suppression is not modulated by affective knowledge. PLoS ONE, 11(4). https://doi.org/10.1371/journal.pone.0150931

Raymond, J. E., \& O’Brien, J. L. (2009). Selective visual attention and motivation: The consequences of value learning in an attentional blink task. Psychological Science, 20(8), 981-988. https://doi.org/10.1111/j.1467-9280.2009.02391.x

Rothkirch, M., Ostendorf, F., Sax, A. L., \& Sterzer, P. (2013). The influence of motivational salience on saccade latencies. Experimental Brain Research, 224(1), 35-47. https://doi.org/10.1007/s00221-012-3284-4

Rothkirch, M., Schmack, K., Schlagenhauf, F., \& Sterzer, P. (2012). Implicit motivational value and salience are processed in distinct areas of orbitofrontal cortex. NeuroImage, 62(3), 1717-1725. https://doi.org/10.1016/j.neuroimage.2012.06.016

Rutherford, H. J. V., O’Brien, J. L., \& Raymond, J. E. (2010). Value associations of irrelevant stimuli modify rapid visual orienting. Psychonomic Bulletin and Review, 17(4), 536-542. https://doi.org/10.3758/PBR.17.4.536

Stein, T. (2019). The breaking continuous flash suppression paradigm: Review, evaluation, and 
outlook. In G. Hesselmann (Ed.), Transitions Between Consciousness and Unconsciousness (pp. 1-38). Routledge.

Stein, T., Awad, D., Gayet, S., \& Peelen, M. V. (2018). Unconscious processing of Facial Dominance: The role of low-level factors in access to awareness. Journal of Experimental Psychology: General, 147(11). https://doi.org/10.1037/xge0000521

Stein, T., Grubb, C., Bertrand, M., Suh, S. M., \& Verosky, S. C. (2017). No impact of affective person knowledge on visual awareness: Evidence from binocular rivalry and continuous flash suppression. Emotion, 17(8). https://doi.org/10.1037/emo0000305

Stein, T., Siebold, A., \& Van Zoest, W. (2016). Testing the idea of privileged awareness of selfrelevant information. Journal of Experimental Psychology: Human Perception and Performance, 42(3). https://doi.org/10.1037/xhp0000197

Stein, T., Sterzer, P., \& Peelen, M. V. (2012). Privileged detection of conspecifics: Evidence from inversion effects during continuous flash suppression. Cognition, 125(1), 64-79. https://doi.org/10.1016/j.cognition.2012.06.005

Tamietto, M., \& De Gelder, B. (2010). Neural bases of the non-conscious perception of emotional signals. Nature Reviews Neuroscience, 11(10), 697-709. https://doi.org/10.1038/nrn2889

Team, J. (2020). JASP (Version 0.12.2).

Tsuchiya, N., \& Koch, C. (2005). Continuous flash suppression reduces negative afterimages. Nature Neuroscience, 8(8), 1096-1101. https://doi.org/10.1038/nn1500

Yang, E., Zald, D. H., \& Blake, R. (2007). Fearful expressions gain preferential access to awareness during continuous flash suppression. Emotion, 7(4), 882-886. https://doi.org/10.1037/1528-3542.7.4.882

Zhan, M., Hortensius, R., \& De Gelder, B. (2015). The body as a tool for anger awarenessdifferential effects of angry facial and bodily expressions on suppression from awareness. PLoS ONE. https://doi.org/10.1371/journal.pone.0139768 